\title{
Non-perturbative Non-integrability of Non-homogeneous Nonlinear Lattices Induced by Non-resonance Hypothesis *
}

\author{
Ken Umeno ${ }^{\dagger}$ \\ Laboratory for Information Representation \\ Frontier Research Program, RIKEN \\ 2-1 Hirosawa, Wako, Saitama 351-01, Japan
}

\begin{abstract}
We have succeeded in applying Ziglin's test on non-integrability to nonhomogeneous nonlinear lattices (Fermi-Pasta-Ulam lattices). By explicit calculations of the eigenvalues of the monodromy matrices concerning the normal variational equations of Lamé type and with the use of the non-resonance hypothesis about the eigenvalues, we obtained a theorem proving the nonexistence of additional analytic conserved quantities other than the Hamiltonian itself for FPU lattices in the low energy limit. Furthermore, after introducing a concept of degree of non-integrability, we have investigated the classification of non-homogeneous nonlinear lattices using a transformation $\Re$ from a non-homogeneous nonlinear lattice to another non-homogeneous nonlinear lattice, which preserves their degree of non-integrability.
\end{abstract}

key words: non-integrability, nonlinear lattices, singularity analysis, monodromy matrices, non-resonance condition

*To appear in PhysicaD

${ }^{\dagger}$ E-mail: chaosken@giraffe.riken.go.jp 


\section{Introduction}

Recently, we know two major sources of detecting non-integrability of Hamiltonian systems. One is Poincaré's theorem 8$]$ of nineteenth century and the other is Ziglin's analysis 19 relatively recently found in the connection with the classical singularity analysis 9 by Fuchs, Kowalevskaya and Painlevé. What matters here is the following thing. The two different methods have significantly different predictive capacities to tell non-integrability. While Poincaré's theorem is asserting the non-existence of additional conserved quantities such as

$$
\Phi_{0}\left(\left\{q_{\nu}\right\}\right)+\mu \Phi_{1}\left(\left\{q_{\nu}, p_{\nu}\right\}\right)+\mu^{2} \Phi_{2}\left(\left\{q_{\nu}, p_{\nu}\right\}\right)+\cdots
$$

for a Hamiltonian system perturbed from an integrable systems

$$
H=H_{0}\left(\left\{q_{\nu}\right\}\right)+\mu H_{1}\left(\left\{q_{\nu}, p_{\nu}\right\}\right)+\mu^{2} H_{2}\left(\left\{q_{\nu}, p_{\nu}\right\}\right)+\cdots
$$

where $\mu$ is a coupling constant whose explicit value is unknown, Ziglin's type theorem can prove the non-existence of additional analytic integral for an explicit Hamiltonian. To illustrate the differences between them more concretely, we can give the following examples. The restricted three-body problem was proved to be non-integrable by Whittaker in 1904[14] based on Poincaré's theorem, while Ziglin's theorem have never proved its non-integrability because of the problem that a Hamiltonian of the restricted threebody problem cannot be defined without the infinitesimal operation $(m \rightarrow 0)$ and we don't have any explicit Hamiltonian which is necessary to perform solid Ziglin's analysis. On the other hand, Poincaré's theorem assumes the condition on the Hessian of Hamiltonian, i.e., the Hessian of Hamiltonian should not vanish

$$
\left|\frac{\partial^{2} H_{0}}{\partial q_{\nu} \partial q_{\lambda}}\right| \neq 0
$$

By this assumption, Poincaré's theorem cannot not tell anything about the nonintegrability of nonlinear lattices consisting of harmonic terms $H_{0}=\frac{1}{2} \sum_{i=1}^{n} q_{i}^{2}$ plus unharmonic terms such as the nonlinear lattices of Fermi-Pasta-Ulam type (FPU lattices). Therefore, in the present paper, we examine the possibility of non-integrability proof of FPU lattices via Ziglin's analysis. It should be also mentioned that Ziglin's analysis have some technical difficulties, as is illustrated later, and no non-integrability proof has been so far studied either for realistic Hamiltonian systems such as the FPU lattices with non-homogeneous potential functions, except few rare instances such as the Henon-Heiles systems with two degrees of freedom[5, 20, 6] or for Hamiltonian systems with many degrees of freedom besides the case of Hamiltonian systems with global and symmetric coupling 13.

The purpose of the present paper is to show that this Ziglin's analysis can be performed for checking the non-integrability of nonlinear lattices including the FPU lattices and to show that these nonlinear lattices have no other analytic conserved quantities.

In Section 2, we show that singularity analysis can be done for a class of FPU lattices by giving the explicit eigenvalues of the monodromy matrices about a special solution in terms of elliptic functions. 
In Section 3, we prove a non-integrability theorem for FPU lattices based on the singularity analysis in Section 2, which shows that each FPU lattice has no additional analytic conserved quantities in the low energy limit. In Section 4 we discuss the classification of non-homogeneous nonlinear lattices via the degree of non-integrability.

In Section 5, we give a brief summary and open questions about the present analysis.

\section{Singularity analysis of nonlinear lattices}

Consider the following one-dimensional lattice:

$$
H=\frac{1}{2} \sum_{i=1}^{n} p_{i}^{2}+\sum_{i=1}^{n+1} v\left(q_{i-1}-q_{i}\right)
$$

If this Hamiltonian admits the reflective invariance, namely, if it is invariant under the involutive symplectic diffeomorphism,

$$
J_{r} H=H
$$

where

$$
J_{r}: \boldsymbol{C}^{2 n} \rightarrow \boldsymbol{C}^{2 n}, J_{r}:\left(q_{1}, \cdots, q_{n}, p_{1}, \cdots, p_{n}\right) \mapsto\left(-q_{1}, \cdots,-q_{n},-p_{1}, \cdots,-p_{n}\right),
$$

the potential function $v(X)$ must satisfy the condition given by

$$
v(X)=v(-X)
$$

In the case of harmonic lattices, we have $v(X)=\frac{1}{2} \mu_{2} X^{2}$. Thus more general nonlinear lattices with the reflection symmetry can be defined by the following polynomial function of even degree in $X$ :

$$
v(X)=\frac{\mu_{2}}{2} X^{2}+\frac{\mu_{4}}{4} X^{4}+\cdots+\frac{\mu_{2 m}}{2 m} X^{2 m} .
$$

In this paper, however, we assume that this potential function is a polynomial function of degree 4, namely, we consider the Fermi-Pasta-Ulam(FPU) lattices 3]

$$
H_{F P U}=\frac{1}{2} \sum_{i=1}^{n} p_{i}^{2}+\frac{\mu_{2}}{2} \sum_{i=1}^{n+1}\left(q_{i-1}-q_{i}\right)^{2}+\frac{\mu_{4}}{4} \sum_{i=1}^{n+1}\left(q_{i-1}-q_{i}\right)^{4} .
$$

Imposing the fixed boundary condition as

$$
q_{0}=q_{n+1}=0, \quad n=\text { odd },
$$

the equations of motion are given by

$$
\begin{aligned}
& \ddot{q}_{1}=-\mu_{2}\left(2 q_{1}-q_{2}\right)-\mu_{4}\left(q_{1}^{3}+\left(q_{1}-q_{2}\right)^{3}\right), \\
& \ddot{q}_{2}=-\mu_{2}\left(2 q_{2}-q_{1}-q_{3}\right)-\mu_{4}\left(\left(q_{2}-q_{1}\right)^{3}+\left(q_{2}-q_{3}\right)^{3}\right), \\
& \vdots \ldots \\
& \vdots \ldots \\
& \ddot{q_{n}}=-\mu_{2}\left(2 q_{n}-q_{n-1}\right)-\mu_{4}\left(\left(q_{n}-q_{n-1}\right)^{3}+q_{n}^{3}\right)
\end{aligned}
$$


Define $M^{\prime}=\left\{(\boldsymbol{q}, \boldsymbol{p}) \in \boldsymbol{C}^{2 n} \mid q_{1} \neq 0\right.$ or $\left.p_{1} \neq 0\right\}, \hat{M}=M^{\prime} / J$ and the canonical projection $\pi: M^{\prime} \rightarrow \hat{M}$. An additional complex analytic integral in $\boldsymbol{C}^{2 n}$, if it exists, also induces the corresponding analytic integral on $\hat{M}$. Therefore it is sufficient to check the non-integrability of the reduced equations of FPU lattices on $\hat{M}$ in order to confirm the non-integrability of the original equations of FPU lattices.

Consider the following one-parameter family of the solution for (2.8)

$$
\Gamma(\epsilon, t): q_{j}=\frac{\left((-1)^{j}-1\right)}{2}(-1)^{\frac{j+1}{2}} C \phi(t)
$$

with the initial condition

$$
\phi(0)=1, \quad \dot{\phi}(0)=0, \quad H_{F P U}(0)=\epsilon .
$$

This is a complex solution in the complex time plane as

$$
q_{1}=C \phi(t), q_{2}=0, q_{3}=-C \phi(t), \cdots, q_{n-1}=0, q_{n}=(-1)^{\frac{n-1}{2}} C \phi(t),
$$

which is depicted in Fig. 1. Thus, the underlying equation of $\phi(t)$ is reduced to the following Hamiltonian system with one degree of freedom:

$$
\ddot{\phi}+2 \mu_{2} \phi+2 \mu_{4} C^{2} \phi^{3}=0,
$$

whose Hamiltonian is given by

$$
H(\phi, \dot{\phi})=\frac{1}{2}(\dot{\phi})^{2}+\mu_{2} \phi^{2}+\frac{\mu_{4} C^{2}}{2} \phi^{4}=\text { Const. }
$$

Then the original total energy $\epsilon$ is written as follows:

$$
\epsilon=H=H(\phi, \dot{\phi}) \frac{n+1}{2} C^{2}=\frac{n+1}{2} C^{2}\left(\mu_{2}+\frac{1}{2} \mu_{4} C^{2}\right)
$$

by the initial condition (2.10). This equation (2.14) enable us to choose $C$ as the following complex quantity:

$$
C=\sqrt{\frac{\sqrt{\mu_{2}^{2}+\frac{4 \epsilon}{n+1} \mu_{4}}-\mu_{2}}{\mu_{4}}} .
$$

Therefore, $H(\phi, \dot{\phi})$ becomes

$$
H(\hat{q}, \hat{p})=\frac{1}{2} \hat{p}^{2}+\mu_{2} \hat{q}^{2}+\frac{\sqrt{\mu_{2}^{2}+\frac{4 \epsilon}{n+1} \mu_{4}}-\mu_{2}}{2} \hat{q}^{4},
$$

where $\hat{q}=\phi, \hat{p}=\dot{\phi}$. By combining (2.13) with (2.14), we obtain the differential equation of $\phi(t)$ as

$$
\frac{1}{2}(\dot{\phi})^{2}=\gamma_{2}\left(1-\phi^{2}\right)+\frac{\gamma_{4}}{2}\left(1-\phi^{4}\right)
$$

with the system parameters

$$
\gamma_{2} \equiv \mu_{2}, \quad \gamma_{4} \equiv \mu_{4} C^{2}
$$


Thus, the phase curve $\Gamma(t, \epsilon)(2.9)$ is given by the elliptic integral

$$
\int_{\phi(0)=1}^{\phi(t)} \frac{d \phi}{\sqrt{2} \sqrt{\gamma_{2}\left(1-\phi^{2}\right)+\frac{\gamma_{4}}{2}\left(1-\phi^{4}\right)}}=t
$$

and the solution of this differential equation (2.17) with the condition

$$
\gamma_{2 m=4} \neq 0
$$

is given explicitly by the formula

$$
\phi(t)=c n(k ; \alpha t),
$$

where

$$
\alpha=\sqrt{2 \gamma_{2}+2 \gamma_{4}}, \quad k=\sqrt{\frac{\gamma_{4}}{2 \gamma_{2}+2 \gamma_{4}}},
$$

$c n(k ; \alpha t)$ is the Jacobi $c n$ elliptic function, and $k$ is the modulus of the elliptic integral, because of the following equalities

$$
\begin{aligned}
& \left(\frac{d \phi}{d t}\right)^{2}=\{-\alpha s n(k ; \alpha t) d n(k ; \alpha t)\}^{2} \\
& =\alpha^{2}\left\{\left(-2 k^{2}+1\right)\left(1-c n^{2}(k ; \alpha t)\right)+k^{2}\left(1-c n^{4}(k ; \alpha t)\right)\right\} \\
& =2 \gamma_{2}\left(1-\phi^{2}\right)+\gamma_{4}\left(1-\phi^{4}\right)
\end{aligned}
$$

in Eq. (2.17). We remark here that the relation

$$
\gamma_{2}+\gamma_{4}=\mu_{2}+C^{2} \mu_{4}=\sqrt{\mu_{2}^{2}+\frac{4 \epsilon}{n+1} \mu_{4}}>0,
$$

holds for $\mu_{4}>0, \mu_{2}>0, \epsilon>0$. This means that the modulus of the elliptic function $k$ satisfies the following relation:

$$
0<k=\frac{1}{\sqrt{2}} \sqrt{1-\frac{1}{\sqrt{1+\frac{4 \epsilon \mu_{4}}{(n+1) \mu_{2}^{2}}}}}<\frac{1}{\sqrt{2}}
$$

and $C$ is a real quantity. On the contrary, if the following relations

$$
\mu_{2}>0, \mu_{4}>0, \epsilon<0
$$

are satisfied, the modulus of the elliptic function $k$ becomes pure imaginary, i.e., $k^{2}<0$. $C$ becomes also pure imaginary as in Fig.1. We note here that even in this unphysical case, there is no barrier to perform the following singularity analysis. However, in the following we shall consider the physical case with the condition as $\mu_{2}>0, \mu_{4}>0, \epsilon>0$. According to the reduction $\pi: M^{\prime} \rightarrow \hat{M}=M^{\prime} / J$ by the reflective symmetry $J$, the solution (2.11) of the induced FPU lattices on $\hat{M}$ for $\mu_{4}>0, \mu_{2} \geq 0$ is a single-valued, meromorphic and doubly periodic function in $t$, having the two fundamental periods in the complex time plane as follows:

$$
T_{1}(\epsilon, \mu)=\frac{2 K(k)}{\alpha}, \quad T_{2}(\epsilon, \mu)=\frac{2 K(k)+2 i K^{\prime}(k)}{\alpha} .
$$


Here $K(k)$ and $K^{\prime}(k)$ are the complete elliptic integrals of the first kind:

$$
K(k)=\int_{0}^{1} \frac{d v}{\sqrt{\left(1-v^{2}\right)\left(1-k^{2} v^{2}\right)}}, \quad K^{\prime}(k)=\int_{0}^{1} \frac{d v}{\sqrt{\left(1-v^{2}\right)\left(1-\left(1-k^{2}\right) v^{2}\right)}} .
$$

Note here that these solutions have a single pole at $t=\tau$, where $\tau=\frac{2 K(k)}{\alpha}+i \frac{K^{\prime}(k)}{\alpha} \quad\left(\bmod T_{1}, T_{2}\right)$ in the parallelogram of each period cell; the phase curves $\Gamma(\epsilon, t)$ are punctured tori. If we consider the solution (2.11) not on the reduced phase space $\hat{M}=M^{\prime} / J$ but on the original phase space $M^{\prime}$, the solution has the following fundamental periods as

$$
T_{1}^{\prime}(\epsilon, \mu)=\frac{4 K(k)}{\alpha}, \quad T_{2}^{\prime}(\epsilon, \mu)=\frac{2 K(k)+2 i K^{\prime}(k)}{\alpha},
$$

which are different from (2.27) and this solution on $M^{\prime}$ has two poles at $t=\tau^{\prime}$, where $\tau^{\prime}=\frac{2 K(k)}{\alpha}+i \frac{K^{\prime}(k)}{\alpha}, \frac{4 K(k)}{\alpha}+i \frac{K^{\prime}(k)}{\alpha} \quad\left(\bmod T_{1}^{\prime}, T_{2}^{\prime}\right)$ in the parallelogram of each periodic cell.

Let us consider the variational equations along the phase curve $\Gamma(\epsilon, t)$ in (2.9) of the general nonlinear lattices with the reflection symmetry. The linearized variational equations are written as follows:

$$
\begin{aligned}
& \dot{\eta}_{j}=\ddot{\xi}_{j}=-\left.\sum_{k=1}^{n} \frac{\partial^{2} V}{\partial q_{k} \partial q_{j}}\right|_{\Gamma} \xi_{k} \\
& =-\left(\gamma_{2}+3 \gamma_{4} c n^{2}(k ; \alpha t)\right)\left(2 \xi_{j}-\xi_{j-1}-\xi_{j+1}\right) \text { for } 1 \leq j \leq n,
\end{aligned}
$$

where $\xi_{0}=\xi_{n+1}=\eta_{0}=\eta_{n+1}=0$ and $\xi_{j}=\delta q_{j}, \eta_{j}=\delta p_{j} \quad(1 \leq j \leq n)$.

If we rewrite these linear variational equations (2.30) in the form of the vector variational equation as

$$
\frac{d^{2}}{d t^{2}} \boldsymbol{\xi}=-\left(\gamma_{2}+3 \gamma_{4} c n^{2}(k ; \alpha t)\right)\left[\begin{array}{ccccc}
2 & -1 & 0 & \ldots & 0 \\
-1 & 2 & -1 & \ldots & 0 \\
0 & -1 & 2 & -1 & \ldots \\
\ldots & \ldots & \ldots & \ldots & \ldots \\
0 & \ldots & 0 & -1 & 2,
\end{array}\right] \boldsymbol{\xi}
$$

and use the fact that the eigenvalues of the $n \times n$ symmetric matrix

$$
\boldsymbol{G}=\left[\begin{array}{ccccc}
2 & -1 & 0 & \cdots & 0 \\
-1 & 2 & -1 & \cdots & 0 \\
0 & -1 & 2 & -1 & \cdots \\
\cdots & \cdots & \cdots & \cdots & \cdots \\
0 & \cdots & 0 & -1 & 2
\end{array}\right]
$$

are obtained as $\left\{4 \sin ^{2}\left(\frac{j \pi}{2(n+1)}\right) \mid 1 \leq j \leq n\right\}$ by a normal orthogonal transformation $\boldsymbol{G} \rightarrow$ $\boldsymbol{O} G \boldsymbol{O}^{-1}$, the variational equations (2.30) can be transformed into the decoupled form:

$$
\ddot{\xi}_{j}^{\prime}(t)=-4 \sin ^{2}\left(\frac{j \pi}{2(n+1)}\right)\left(\gamma_{2}+3 \gamma_{4} c n^{2}(k ; \alpha t)\right) \xi_{j}^{\prime}(t) \quad(1 \leq j \leq n)
$$


where $\boldsymbol{\xi}^{\prime}=\boldsymbol{O} \boldsymbol{\xi}$. Clearly, these equations are regarded as the vector form of Hill's equations[]]

$$
\frac{d^{2} \boldsymbol{\xi}^{\prime}}{d t^{2}}+\boldsymbol{A}(t) \boldsymbol{\xi}^{\prime}=0, \quad \boldsymbol{A}(t+T)=\boldsymbol{A}(t),
$$

where $T=T_{1}, T_{2}$. For $j=\frac{n+1}{2}$, the relation

$$
\xi_{\frac{n+1}{2}}^{\prime}=\sqrt{\frac{2}{n+1}}\left(\xi_{1}-\xi_{3}+\xi_{5}+\cdots+(-1)^{\frac{n-1}{2}} \xi_{n}\right)
$$

holds. The corresponding variational equation

$$
\ddot{\xi}_{\frac{n+1}{2}}^{\prime}=-2\left(\gamma_{2}+3 \gamma_{4} c n^{2}(k ; \alpha t)\right) \xi_{\frac{n+1}{2}}^{\prime}(t)
$$

has a time-dependent integral $I(\boldsymbol{\xi}, \dot{\boldsymbol{\xi}} ; t) \equiv I(\boldsymbol{\xi}, \boldsymbol{\eta} ; t)$ derived from the variational operation $\boldsymbol{D}$ applied to the Hamiltonian (2.6):

$$
\begin{aligned}
& I(\boldsymbol{\xi}, \boldsymbol{\eta} ; t)=\boldsymbol{D} H \equiv\left(\boldsymbol{\eta} \cdot \frac{\partial}{\partial \boldsymbol{p}}+\boldsymbol{\xi} \cdot \frac{\partial}{\partial \boldsymbol{q}}\right) H=\boldsymbol{\eta} \cdot \boldsymbol{p}+\boldsymbol{\xi} \cdot \boldsymbol{V}_{\boldsymbol{q}} \\
& =C \dot{\phi}\left(\eta_{1}-\eta_{3}+\eta_{5}+\cdots+(-1)^{\frac{n-1}{2}} \eta_{n}\right) \\
& +2\left(C \gamma_{2} c n(k ; \alpha t)+C \gamma_{4} c n^{3}(k ; \alpha t)\right)\left(\xi_{1}-\xi_{3}+\xi_{5}+\cdots+(-1)^{\frac{n-1}{2}} \xi_{n}\right),
\end{aligned}
$$

because

$$
\begin{aligned}
& \frac{1}{C} \frac{d I}{d t}=\dot{\phi}\left(\ddot{\xi}_{1}-\ddot{\xi}_{3}+\cdots+(-1)^{\frac{n-1}{2}} \ddot{\xi}_{n}\right) \\
& +2 \dot{\phi}\left(\gamma_{2}+3 \gamma_{4} c n^{2}(k ; \alpha t)\right)\left(\xi_{1}-\xi_{3}+\cdots+(-1)^{\frac{n-1}{2}} \xi_{n}\right)=0 .
\end{aligned}
$$

Eq. (2.36) is called the tangential variational equation. On the other hands, a $(2 n-2)$ dimensional normal variational equation(NVE) is defined by the equations (2.33) with the tangential variational equation (2.36) removed as follows:

$$
\begin{aligned}
& \dot{\eta}_{j}^{\prime}=-4 \sin ^{2}\left(\frac{j \pi}{2(n+1)}\right)\left(\gamma_{2}+3 \gamma_{4} c n^{2}(k ; \alpha t)\right) \xi_{j}^{\prime}, \\
& \dot{\xi}_{j}^{\prime}=\eta_{j}^{\prime}
\end{aligned}
$$

for $1 \leq j\left(\neq \frac{n+1}{2}\right) \leq n$.

Let us consider the monodromy matrices $g$ defined by the analytic continuation of the solution $\zeta^{\prime}(t)=\left(\xi_{1}^{\prime}(t), \eta_{1}^{\prime}(t), \cdots, \hat{\xi}_{\frac{n+1}{2}}^{\prime}(t), \hat{\eta}_{\frac{n+1}{2}}^{\prime}(t), \cdots, \xi_{n}^{\prime}(t), \eta_{n}^{\prime}(t)\right)$ of the NVE (2.39) along the periodic orbits in the phase curves $\Gamma(\epsilon, t)$ as follows:

$$
\boldsymbol{\zeta}^{\prime}\left(T_{1}\right)=g_{1} \boldsymbol{\zeta}^{\prime}(0), \quad \boldsymbol{\zeta}^{\prime}\left(T_{2}\right)=g_{2} \boldsymbol{\zeta}^{\prime}(0) .
$$

The periods in Eqs. (2.40) are $T_{1}, T_{2}$ in Eqs. (2.27), respectively. These two fundamental periods $T_{1}$ and $T_{2}$ naturally form the parallelogram, whose associate monodromy matrices are given by $g_{1} g_{2} g_{1}^{-1} g_{2}^{-1}\left(\equiv g_{*}\right)$. These monodromy matrices are naturally endowed with the symplectic structure and the pairing properties of the eigenvalues, namely $\left\{\sigma_{1}, \sigma_{1}^{-1}, \sigma_{2}, \sigma_{2}^{-1}, \cdots, \sigma_{n}, \sigma_{n}^{-1}\right\}$. In general, the explicit calculation of the eigenvalues of the monodromy matrices is an unsuccessful business except rare cases such as Hamiltonian systems with homogeneous polynomial functions [17], Riemann's equation, and the

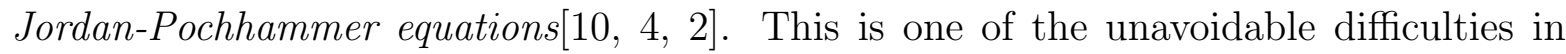
performing Ziglin's analysis of general dynamical systems. In our case, however, we can 
compute explicitly the eigenvalues of the commutator $g_{*}=g_{1} g_{2} g_{1}^{-1} g_{2}^{-1}$ by using the fact that the normal variational equation (2.39) happens to be in a class of Lamé equations 15.

$$
\frac{d^{2} y}{d t^{2}}-\left(E_{1} s n^{2}(k ; \alpha t)+E_{2}\right) y=0
$$

where $E_{1}$ and $E_{2}$ are constants, and the eigenvalues $\sigma$ of the commutator $g_{*}=g_{1} g_{2} g_{1}^{-1} g_{2}^{-1}$ are known [15] to be determined by the indicial equation

$$
\Delta^{2}-\Delta-\left(\alpha^{2} k^{2}\right) E_{1}=0, \quad \sigma=\exp (2 \pi i \Delta)
$$

with the singular point (pole) $\tau$ located at the center of the parallelogram:

$$
\tau=\frac{T_{1}+T_{2}}{2}=\frac{2 K(k)+i K^{\prime}(k)}{\alpha} .
$$

If we apply the indicial equation(2.42) to the normal variational equations $(2.39)$ of the FPU lattices, the exponents of the eigenvalues of $g_{*}$ are given by

$$
\Delta^{2}-\Delta-12 \frac{\gamma_{4}}{\alpha^{2} k^{2}} \sin ^{2}\left(\frac{j \pi}{2(n+1)}\right)=0
$$

Noting

$$
\frac{\gamma_{4}}{\alpha^{2} k^{2}}=1
$$

from (2.22), we finally obtain the eigenvalues of the commutator as

$$
\begin{aligned}
\sigma_{j} & =\exp \left(2 \pi i \frac{1 \pm \sqrt{1+48 \sin ^{2} \frac{j \pi}{2(n+1)}}}{2}\right) \\
& =-\exp \left( \pm \pi i \sqrt{25-24 \cos \left(\frac{j \pi}{n+1}\right)}\right)
\end{aligned}
$$

for $1 \leq j\left(\neq \frac{n+1}{2}\right) \leq n$, because there is only one pole singularity inside the parallelogram formed by counterclockwise closed loop of the monodromy group $g_{1} g_{2} g_{1}^{-1} g_{2}^{-1}$. Even for more general nonlinear lattices given by potential functions (2.5) of degree $2 m$ we can also compute the phase factors. Let us consider singular solutions $\phi(t)$ and $\xi_{j}(t)$ whose singularities are located at $t=\tau$ in the complex time plane as follows:

$$
\begin{aligned}
& \phi(t)=C^{\prime}(t-\tau)^{\beta}, \quad \beta<0 \\
& \xi_{j}(t)=C^{\prime \prime}(t-\tau)^{\nu_{j}},
\end{aligned}
$$

where $C^{\prime}$ is a particular constant, $C^{\prime \prime}$ is an arbitrary constant. Thus, we can easily obtaine the formulas the relations for $\beta, C^{\prime}, \nu_{j}$ as

$$
\begin{aligned}
& \beta=\frac{-1}{m-1}, \\
& \left(C^{\prime}\right)^{2 m-2}=-\frac{\beta(\beta-1)}{2 \gamma_{2 m}}, \\
& \nu_{j}^{2}-\nu_{j}-2 \frac{m(2 m-1)}{(m-1)^{2}} \sin ^{2}\left(\frac{j \pi}{2(n+1)}\right)=0
\end{aligned}
$$


by the equations of motion. With the use of the fact that the phase factor is obtained by means of the analytic continuation along the closed loop around the singular point $t^{\nu_{j}}$, we obtain the phase factors as

$$
\begin{gathered}
=\exp \left\{2 \pi i \frac{\left.1 \pm \sqrt{1+8 \frac{m(2 m-1)}{(m-1)^{2}} \sin ^{2}\left(\frac{j \pi}{2(n+1)}\right.}\right)}{2}\right\} \\
=-\exp \left\{ \pm \pi i \sqrt{1+8 \frac{m(2 m-1)}{(m-1)^{2}} \sin ^{2}\left(\frac{j \pi}{2(n+1)}\right)}\right\}
\end{gathered}
$$

where $2 m$ is the degree of the potential polynomial of $v(X)$ in (2.5). It is easy to confirm that in the case that $2 m=4$, the formula (2.49) recover the eigenvalues (2.46) of the monodromy matrices obtained from the Jacobi elliptic function. However, in the other case that $2 m>4$, we cannot compute the eigenvalues of monodromy matrices only from the phase factors in general, because the normal variational equations do not belong to a class of Lamé equations. To summarize, we can say that it is essential to perform the singularity analysis towards non-homogeneous nonlinear lattices using the explicit eigenvalues of the monodromy matrices that the special solutions can be written in terms of elliptic functions and the normal variational equations are in a type of Lamé equations.

\section{Non-integrability theorem}

\subsection{Non-integrability proof in the integrable limit}

The chief purpose of the present section is to give a theorem telling the non-integrability by using the monodromy matrices obtained in Section 2. We consider monodromy matrices $g$. If the eigenvalues $\left\{\sigma_{1}, \sigma_{1}^{-1}, \cdots, \sigma_{n}, \sigma_{n}^{-1}\right\}$ of monodromy matrices $g$ do not satisfy the following relation

$$
\sigma_{1}^{l_{1}} \sigma_{2}^{l_{2}} \cdots \sigma_{n}^{l_{n}}=1
$$

for any set of integers $\left\{l_{1}, \cdots, l_{n}\right\}$ except the trivial case $l_{1}=l_{2}=\cdots=l_{n}=0$, we call the monodromy matrices $g$ non-resonant. It is already known that the existence of a non-resonant monodromy matrix is a basic assumption in order to perform Ziglin's analysis 19. Moreover, if there are straight-line solutions such as (2.11) whose monodromy matrices are non-resonant and if the variational equations can be diagonalized into a decoupled form like (2.33), Ziglin's theorem can be generalized to Yoshida's theorem for Hamiltonian systems composed by kinetic energy terms and potential energy terms 16.

Yoshida's theorem asserts the following in terms of two different monodromy matrices $\left\{g_{a}, g_{b}\right\}$ : Suppose that there exists an additional complex analytic integral, which is holomorphic along the solution (2.9), and that one of the monodromy matrices $g_{a}$ is non-resonant. Then it is necessary that one of the following two cases, namely,

(I) $g_{b}\left(\lambda_{j}\right)$ must preserve the eigendirection of $g_{a}\left(\lambda_{j}\right)$, i.e., $g_{a}\left(\lambda_{j}\right)$ must commute with $g_{b}\left(\lambda_{j}\right)$ 
(II) $g_{b}\left(\lambda_{j}\right)$ must permute the eigendirection of $g_{a}\left(\lambda_{j}\right)$, i.e., $g_{b}\left(\lambda_{j}\right)$ is written by $\left[\begin{array}{cc}0 & \beta \\ -\frac{1}{\beta} & 0\end{array}\right]$ in the base of $g_{a}$ having the eigenvalues $i$ and $-i$

for some suffix $j$, at least occurs for any other monodromy matrix $g_{b}$ represented in the basis of $g_{a}$. As for the non-resonance condition of the monodromy matrix $g_{*}=$ $g_{1} g_{2} g_{1}^{-1} g_{2}^{-1}$, the following lemma is already known:

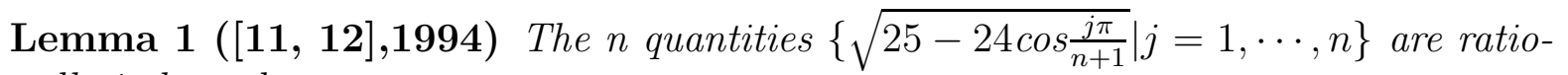
nally independent.

We remark that the rational independency of the set

$\left\{\sqrt{25-24 \cos \frac{j \pi}{n+1}} \mid j=1, \cdots n\right\}$ guarantees that the commutator $g_{*}=g_{1} g_{2} g_{1}^{-1} g_{2}^{-1}$ whose eigenvalues are given in Eq. (2.46) is non-resonant.

For connection with the algebraic number theory on the cyclotomic field $\boldsymbol{Q}\left(\exp \left(\frac{\pi i}{n+1}\right)\right)$ over $\boldsymbol{Q}$ see Ref. [12]. Thus, we can regard that the non-resonance hypothesis holds for the monodromy matrix $g_{*}$. Now, using Yoshida's theorem and the variational analysis in the former section, we obtain a theorem which proves the non-integrability of a FPU lattice for an arbitrary set of the system parameters $\left\{n, \mu_{2}, \mu_{4} \mid n(\geq 3)\right.$ : odd, $\left.\mu_{2}>0, \mu_{4}>0\right\}$ as follows:

Theorem 1 A FPU lattice (2.0) which is characterized by an arbitrary set of the system parameters $\left\{n, \mu_{2}, \mu_{4} \mid n \geq 3, n:\right.$ odd $\left., \mu_{2}>0, \mu_{4}>0\right\}$ has no analytic first integrals besides the Hamiltonian itself for fully small energy $\epsilon(\approx 0)$.

(Proof of Theorem 1)

From the non-resonance hypothesis on $g_{*}$, we can apply the Yoshida theorem 16 to the FPU lattices. According to the above argument, if we prove that at least one monodromy matrix $g_{s} \in\left\{g_{1}, g_{2}\right\}$ does not have the following properties

(a) $g_{s}\left(\lambda_{j}\right)$ preserves the eigendirection of $g_{*}\left(\lambda_{j}\right)$ and

(b) $g_{s}\left(\lambda_{j}\right)$ permutes the eigendirection of $g_{*}\left(\lambda_{j}\right)$ (the eigenvalues of $g_{s}\left(\lambda_{j}\right)$ are $\left.i,-i\right)$,

at once for any suffix $j(1 \leq j(\neq n+1 / 2) \leq n)$ of $g_{s}\left(\lambda_{j}\right)$, then the FPU lattices [3] are concluded to have no other analytic conserved quantities besides the Hamiltonian itself, i.e., the assertion of the present theorem holds. In the following, we will show that $g_{1}\left(\lambda_{j}\right)$ for any $j\left(\neq \frac{n+1}{2}\right)$ has neither the property (a) and the property (b).

When we take the limit $\epsilon \rightarrow 0$, the relations

$$
\gamma_{4}=\mu_{4} C^{2}, \cdots, \gamma_{2 m}=\mu_{2 m} C^{2 m-2} \rightarrow 0, \quad \alpha \rightarrow \sqrt{2 \mu_{2}}, \quad k \rightarrow 0
$$

hold and we can compute the periods of the monodromy matrices $g_{1}, g_{2}$ in the limit as

$$
T_{1} \rightarrow \frac{1}{\sqrt{2 \gamma_{2}}} \pi, \quad T_{2} \rightarrow \frac{1}{\sqrt{2 \gamma_{2}}} \pi+i \infty
$$


by the formula (2.27). To compute the monodromy matrix $g_{1}\left(\lambda_{j}\right)$ in this limit, we rewrite the variational equations (2.39) in terms of the modulus of the elliptic integral $k$ as follows

$$
\ddot{\xi}_{j}^{\prime}+4 \mu_{2} \sin ^{2}\left(\frac{j \pi}{2(n+1)}\right)\left(1+\frac{2 k^{2}}{1-2 k^{2}} c n^{2}(k ; \alpha t)\right) \xi_{j}^{\prime}=0, \quad j=1, \cdots, n
$$

by virtue of equations (2.15) and (2.22). For arbitrary $k \in[0,1]$, we have a fundamental system of solutions as $\left\{\xi_{j}^{a}(k ; t), \xi_{j}^{b}(k ; t)\right\}$ of $(3.4)$ satisfying

$$
\begin{array}{ll}
\xi_{j}^{a}(k ; 0)=1, & \left.\frac{d}{d t} \xi_{j}^{a}(k ; t)\right|_{t=0}=\dot{\xi}_{j}^{a}(k ; 0)=0 \\
\xi_{j}^{b}(k ; 0)=0, & \left.\frac{d}{d t} \xi_{j}^{b}(k ; t)\right|_{t=0}=\dot{\xi}_{j}^{b}(k ; 0)=1 .
\end{array}
$$

Because $c n^{2}(k ; \alpha t)$ in Eqs. (3.4) has the following Taylor expansions [1] at $\kappa \equiv k^{2}=0$ :

$$
c n^{2}(k ; \alpha t)=\cos ^{2}\left(\frac{\alpha \pi}{2 K} t\right)-\frac{1}{2} \kappa \sin ^{2}\left(\frac{\alpha \pi}{2 K} t\right) \cos ^{2}\left(\frac{\alpha \pi}{2 K} t\right)+\cdots
$$

and analytic in $\kappa$ at $\kappa=0$, the fundamental system of solutions $\left\{\xi^{a}, \xi^{b}\right\}$ are also analytic in $\kappa$ at $\kappa=0$ [6] as

$$
\begin{aligned}
& \xi_{j}^{a}(k ; t)=\xi_{j, 0}^{a}(t)+\xi_{j, 1}^{a}(t) \kappa+\xi_{j, 2}^{a}(t) \kappa^{2}+\cdots \\
& \xi_{j}^{b}(k ; t)=\xi_{j, 0}^{b}(t)+\xi_{j, 1}^{b}(t) \kappa+\xi_{j, 2}^{a}(t) \kappa^{2}+\cdots
\end{aligned}
$$

where the unperturbed parts $\left\{\xi_{j, 0}^{a}, \xi_{j, 0}^{b}\right\}$ are obtained by

$$
\begin{aligned}
& \xi_{j, 0}^{a}(t)=\cos \left(2 \sin \left(\frac{j \pi}{2(n+1)}\right) \sqrt{\mu_{2}} t\right), \\
& \xi_{j, 0}^{b}(t)=\frac{1}{2 \sqrt{\mu_{2}} \sin \left(\frac{j \pi}{2(n+1)}\right)} \sin \left(2 \sin \left(\frac{j \pi}{2(n+1)}\right) \sqrt{\mu_{2}} t\right)
\end{aligned}
$$

from the initial conditions (3.5). The monodromy matrix $g_{1}\left(\lambda_{j}\right)$ is given by

$$
g_{1}\left(\lambda_{j}\right)=\left[\begin{array}{cc}
\xi_{j}^{a}\left(k ; T_{1}\right) & \xi_{j}^{b}\left(k ; T_{1}\right) \\
\dot{\xi}_{j}^{a}\left(k ; T_{1}\right) & \dot{\xi}_{j}^{b}\left(k ; T_{1}\right)
\end{array}\right]
$$

and with the use of the fact that the fundamental system of solutions (3.8) are analytic in $\kappa$ at $\kappa=0$, we show that $g_{1}\left(\lambda_{j}\right)$ for $j=1, \cdots, n$ have also the Taylor expansions as

$$
g_{1}\left(\lambda_{j}\right)=g_{1,0}\left(\lambda_{j}\right)+g_{1,1}\left(\lambda_{j}\right) \kappa+g_{1,2}\left(\lambda_{j}\right) \kappa^{2}+\cdots
$$

Thus now, when we consider the low energy limit $(\epsilon \rightarrow 0, \kappa \rightarrow 0)$, we obtain the formula

$$
g_{1}\left(\lambda_{j}\right) \rightarrow\left[\begin{array}{cc}
\cos \left(\sqrt{2} \pi \sin \left(\frac{j \pi}{2(n+1)}\right)\right) & \frac{1}{2 \sin \left(\frac{j \pi}{2(n+1)}\right) \sqrt{\mu_{2}}} \sin \left(\sqrt{2} \pi \sin \left(\frac{j \pi}{2(n+1)}\right)\right) \\
-2 \sin \left(\frac{j \pi}{2(n+1)}\right) \sqrt{\mu_{2}} \sin \left(\sqrt{2} \pi \sin \left(\frac{j \pi}{2(n+1)}\right)\right) & \cos \left(\sqrt{2} \pi \sin \left(\frac{j \pi}{2(n+1)}\right) \pi\right)
\end{array}\right] .
$$

This means that the eigenvalues of $g_{1}\left(\lambda_{j}\right)$ tend to

$$
\left\{\operatorname { e x p } \left(i \pi\left(\sqrt{2} \sin \left(\frac{j \pi}{2(n+1)}\right)\right), \exp \left(-i \pi\left(\sqrt{2} \sin \left(\frac{j \pi}{2(n+1)}\right)\right)\right\}\right.\right.
$$


and $g_{1}\left(\lambda_{j}\right)$ for any $j\left(\neq \frac{n+1}{2}\right)$ does not have the property of $(\mathrm{b})$.

Now assume that $g_{2}\left(\lambda_{j}\right)$ for some $j\left(\neq \frac{n+1}{2}\right)$ has the property of $(\mathrm{a})$. Then if $g_{1}\left(\lambda_{j}\right)$ as well as $g_{2}\left(\lambda_{j}\right)$ has also the property of (a), we have

$$
g_{*}\left(\lambda_{j}\right)=g_{1}\left(\lambda_{j}\right) g_{2}\left(\lambda_{j}\right) g_{1}^{-1}\left(\lambda_{j}\right) g_{2}^{-1}\left(\lambda_{j}\right)=\boldsymbol{i d},
$$

where $\boldsymbol{i d}$ denotes the $2 \times 2$ identity matrix. This relation 3.13 means that $g_{1}\left(\lambda_{j}\right)$ and $g_{2}\left(\lambda_{j}\right)$ commute each other and clear contradicts the non-resonance hypothesis of $g_{*}$. Consider the other case where $g_{1}\left(\lambda_{j}\right)$ has the property of (a) and $g_{2}\left(\lambda_{j}\right)$ does not have the property of (a). However, in the representation of $g_{1}\left(\lambda_{j}\right)$ and $g_{2}\left(\lambda_{j}\right)$ in the basis of $g_{*}\left(\lambda_{j}\right)$ as

$$
g_{1}\left(\lambda_{j}\right)=\left[\begin{array}{cc}
\mu & 0 \\
0 & \frac{1}{\mu}
\end{array}\right], \quad g_{2}\left(\lambda_{j}\right)=\left[\begin{array}{ll}
a & b \\
c & d
\end{array}\right](a d-b c=1),
$$

the following relation

$$
g_{*}\left(\lambda_{j}\right)=g_{1}\left(\lambda_{j}\right) g_{2}\left(\lambda_{j}\right) g_{1}^{-1}\left(\lambda_{j}\right) g_{2}^{-1}\left(\lambda_{j}\right)=\left[\begin{array}{cc}
a d-\mu^{2} b c & a b\left(\mu^{2}-1\right) \\
c d\left(\frac{1}{\mu^{2}}-1\right) & a d-\frac{b c}{\mu^{2}}
\end{array}\right]
$$

must be satisfied. Since $g_{*}\left(\lambda_{j}\right)$ is assumed to have a diagonal representation as $g_{*}\left(\lambda_{j}\right)=$ $\operatorname{diag}\left[\sigma_{j}, \sigma_{j}^{-1}\right]$ and from the relation (3.15), we obtain

$$
a=0, \quad d=0, \quad b c=-1,
$$

when $g_{*}\left(\lambda_{j}\right) \neq \boldsymbol{i} \boldsymbol{d} ; g_{2}\left(\lambda_{j}\right)$ must have the property of $(\mathrm{b})$.

Therefore, in the basis of $g_{*}\left(\lambda_{j}\right)$, we have

$$
g_{1}\left(\lambda_{j}\right)=\left[\begin{array}{cc}
\mu & 0 \\
0 & \frac{1}{\mu}
\end{array}\right] \text { and } g_{2}\left(\lambda_{j}\right)=\left[\begin{array}{cc}
0 & \beta \\
-\frac{1}{\beta} & 0
\end{array}\right] .
$$

These relations (3.17) result in

$$
\left[\begin{array}{cc}
\sigma_{j} & 0 \\
0 & \sigma_{j}^{-1}
\end{array}\right]=g_{*}\left(\lambda_{j}\right)=g_{1}\left(\lambda_{j}\right) g_{2}\left(\lambda_{j}\right) g_{1}^{-1}\left(\lambda_{j}\right) g_{2}^{-1}\left(\lambda_{j}\right)=g_{1}^{2}\left(\lambda_{j}\right),
$$

where $\sigma_{j}=-\exp \left\{\pi i \sqrt{25-24 \cos \frac{j \pi}{n+1}}\right\}$. However, the relation (3.18) causes again a contradiction with the fact that the eigenvalues of $g_{1}^{2}\left(\lambda_{j}\right)$ approach to

$$
\left\{\operatorname { e x p } \left(i \pi\left(2 \sqrt{2} \sin \left(\frac{j \pi}{2(n+1)}\right)\right), \exp \left(-i \pi\left(2 \sqrt{2} \sin \left(\frac{j \pi}{2(n+1)}\right)\right)\right\}\right.\right.
$$

in the limit $\epsilon \rightarrow 0$ and there is a difference between the eigenvalues of $g_{*}\left(\lambda_{j}\right)$ and the eigenvalues of $g_{1}^{2}\left(\lambda_{j}\right)$ as will be shown in the Appendix.

We have seen that $g_{1}\left(\lambda_{j}\right)$ for any $j\left(\neq \frac{n+1}{2}\right)$ has neither the property of (a) or the property of (b). Now the theorem holds. ( End of proof of Theorem $\mathbb{1}$ ) 


\section{Classification of non-homogeneous nonlinear lat- tices}

Here in the present section, we consider the classification of these non-integrable nonlinear lattices via the degree of non-integrability. We define the degree of non-integrability as follows:

Definition 1 We write a relation

$$
\{H, \epsilon\} \sim\left\{H^{\prime}, \epsilon^{\prime}\right\}
$$

if and only if Hamiltonian systems with $n$ degrees of freedom $H$ and $H^{\prime}$ have the same number of additional analytic integrals which are functionally independent together with the Hamiltonians $H$ and $H^{\prime}$ respectively. Here $\epsilon\left(\epsilon^{\prime}\right)$ denotes the total energy of the Hamiltonian systems $H\left(H^{\prime}\right)$.

If we associate the positive integer

$$
\varrho=2^{r} \cdot 3^{n-r-1}
$$

with a Hamiltonian system $H$ with $n$ degrees of freedom which has $r$ additional analytic integrals which are functionally independent together with the Hamiltonian $H$, we can classify Hamiltonian systems via the degree of non-integrability as follows:

$$
\{H, \epsilon\} \sim\left\{H^{\prime}, \epsilon^{\prime}\right\} \Longleftrightarrow \varrho(\{H, \epsilon\})=\varrho\left(\left\{H^{\prime}, \epsilon^{\prime}\right\}\right)
$$

For a non-homogeneous nonlinear lattice

$$
H_{\mu_{2}, \mu_{4}, \cdots, \mu_{2 m}}(\boldsymbol{q}, \boldsymbol{p} ; t)=\frac{1}{2} \sum_{i=1}^{n} p_{i}^{2}+\sum_{k=1}^{m} \frac{\mu_{2 k}}{2 k} \sum_{i=1}^{n}\left(q_{i-1}-q_{i}\right)^{2 k}
$$

of degree $2 m$, consider the following transformation $\Re$ from a non-homogeneous nonlinear lattice to another non-homogeneous nonlinear lattice:

Definition 2 We call a transformation $\Re$ from $H_{\mu_{2}, \mu_{4}, \cdots, \mu_{2 m}}(\boldsymbol{q}, \boldsymbol{p} ; t)$ to $H_{\mu_{2}^{\prime}, \mu_{4}^{\prime}, \cdots, \mu_{2 m}^{\prime}}\left(\boldsymbol{q}^{\prime}, \boldsymbol{p}^{\prime} ; t^{\prime}\right)$ a homogenizer if the relations

$$
\begin{aligned}
& t^{\prime}=\alpha t, \quad \boldsymbol{q}^{\prime}=\frac{1}{\alpha^{\frac{1}{m-1}}} \boldsymbol{q}, \quad \boldsymbol{p}^{\prime}=\frac{1}{\alpha^{\frac{m}{m-1}}} \boldsymbol{p} \\
& \mu_{2}^{\prime}=\frac{1}{\alpha^{2}} \mu_{2}, \quad \mu_{4}^{\prime}=\frac{1}{\alpha^{2 \frac{m-2}{m-1}}} \mu_{4}, \quad \cdots, \quad \mu_{2 \nu}^{\prime}=\frac{1}{\alpha^{2 \frac{m-\nu}{m-1}}}, \quad \cdots, \quad \mu_{2 m}^{\prime}=\mu_{2 m},
\end{aligned}
$$

are satisfied where $\alpha$ is a real quantity larger than unity $(1<\alpha<\infty)$.

Remark 1

The transformation $\Re$ in Eqs. (4.5) can be regarded as a generalization of the scaling transformation for homogeneous nonlinear lattices $H_{\mu_{2}=0, \cdots, \mu_{2 m-2}=0, \mu_{2 m}}$. Moreover, the equations of motion itself remain invariant under the transformation $\Re$, while a Hamiltonian is changed according to the variations of coupling constants $\left\{\mu_{2 \gamma} \mid 1 \leq \gamma<m\right\}$. Thus, we can say that $\Re$ preserves the degree of non-integrability as

$$
\{\Re H, \Re \epsilon\} \sim\{H, \epsilon\} .
$$


Remark 2

The operation $\Re$ corresponds to a kind of renormalization group along the time axis from the view point of the coarse graining in statistical physics and it is easy to see that the fixed point of $\Re$ is the homogeneous nonlinear lattice $H^{*}$, i.e.,

$$
H^{*}=\Re H^{*},
$$

where

$$
\begin{aligned}
& H^{*}=\lim _{l \rightarrow \infty} \Re^{l} H \\
& =\frac{1}{2} \sum_{i=1}^{n} p_{i}^{2}+\frac{\mu_{2 m}}{2 m} \sum_{i=1}^{n}\left(q_{i-1}-q_{i}\right)^{2 m}
\end{aligned}
$$

and $\Re^{l}$ denotes $l$ iterations of the operation $\Re$. This is the reason why the $\Re$ is called a homogenizer here.

Remark 3

Under the transformation $\Re$, the total energy $\epsilon$ is changed into $\epsilon^{\prime}$ by the formula

$$
\epsilon^{\prime}=\Re \epsilon=\frac{1}{\alpha^{\frac{2 m}{m-1}}} \epsilon .
$$

By the above remarks, we can conclude that

$$
\left\{H_{\frac{1}{\alpha^{2 l}} \mu_{2}, \mu_{4}}, \frac{1}{\alpha^{4 l}} \epsilon\right\} \sim\left\{H_{\mu_{2}, \mu_{4}}, \epsilon\right\}
$$

for an arbitrary FPU lattice $\left\{H_{\mu_{2}, \mu_{4}}, \epsilon\right\}$. If we take the limit $\alpha^{l} \rightarrow \infty$ preserving the relation $\frac{\epsilon}{\alpha^{4 l}} \approx O(1)$, L.H.S. of (4.10) approaches a homogeneous nonlinear lattice of degree 4, which means that the degree of non-integrability of a homogeneous nonlinear lattice is the same as the degree of non-integrability of a FPU lattice in the high energy limit $\epsilon \rightarrow \infty$. In relation to the non-integrability of the homogeneous nonlinear lattice, there is a known result by Yoshida 17] stating as follows:

Considering $n$ number of distinct exponents

$$
\Delta_{j} \equiv \sqrt{1+\frac{8 m(2 m-1)}{(m-1)^{2}} \sin ^{2}\left(\frac{j \pi}{2(n+1)}\right)} \quad(\text { for } \quad j=1, \cdots, n),
$$

or the $n$ representative of the Kowalevski exponents [18] of the homogeneous nonlinear lattice of degree $2 \mathrm{~m}$. If the n quantities $\left\{\Delta_{j}\right\}$ in (4.11) are rationally independent, then the homogeneous nonlinear lattices have no analytic first integrals except the Hamiltonian itself for $2 m(\geq 4)$ and for odd $n(\geq 3)$.

Note that the $n$ quantities (4.11) equal the phase factors (2.49) of the eigenvalues of the commutator $g_{*}$. Thus, the non-resonance hypothesis proving the non-integrability in the low energy limit $\epsilon \rightarrow 0$ induces also the non-integrability of nonlinear lattices with a nonhomogeneous potential function in the high energy limit $\epsilon \rightarrow \infty$. The reason for this uniqueness of the non-resonance hypothesis lies in the fact that after a conformal mapping $z=\phi^{4}(t)$, the closed loop of the commutator $g_{1} g_{2} g_{1}^{-1} g_{2}^{-1}$ becomes a homotopy 
equivalent loop which corresponds to Yoshida's construction of the monodromy matrix $G=\left(G_{0} G_{1}\right)^{16}$ giving the same non-resonance hypothesis in terms of Kowalevski exponents. Here, $G_{0}$ corresponds to a counterclockwise closed loop around the singularity $z=0$ and $G_{1}$ corresponds to another counterclockwise closed loop around the singularity $z=1$ in the complex $z$ plane, where $z$ satisfies the following Gauss hyper geometric equation:

$$
z(1-z) \frac{d^{2} \xi_{j}^{\prime}}{d z^{2}}+\left[\frac{3}{4}-\frac{5}{4} z\right] \frac{d \xi^{\prime}}{d z}+\frac{3}{2} \gamma_{4} \sin ^{2}\left(\frac{j \pi}{2(n+1)}\right) \xi_{j}^{\prime}=0
$$

into which the conformal mapping $z=\phi^{4}$ transforms the variational Eq. (2.33) with the condition $\gamma_{2}=0$ and $G_{0}, G_{1}$ are the two fundamental monodromy groups of the Gauss hyper geometric equation. See also Fig. 3.

So far, we have succeeded in checking the non-integrability of the FPU lattices both in the low energy limit and in the high energy limit from a single non-resonance hypothesis. However, there still remain some problems about the integrability of FPU lattices for a finite energy $0<\epsilon<\infty$. To consider the problem, we introduce a real parameter

$$
\chi=\frac{\epsilon \mu_{4}}{(n+1) \mu_{2}^{2}}>0
$$

where $\chi$ is found to be a dimensionless parameter, because

$$
\text { Dimension of } \chi=\frac{\left[\frac{\mathrm{L}^{2}}{\mathrm{MT}^{2}}\right]\left[\frac{1}{\mathrm{MT}^{2} \mathrm{~L}^{2}}\right]}{[1]\left[\frac{1}{\mathrm{M}^{2} \mathrm{~T}^{4}}\right]}=[1]
$$

by the standard unit system as $[\mathrm{M}]=[\mathrm{kg}],[\mathrm{T}]=[\mathrm{sec}],[\mathrm{L}]=[\mathrm{m}]$. We remark here that $\chi$ is invariant under the transformation $\Re$ preserving the degree of non-integrability. This means that it is sufficient for us to classify the degree of non-integrability of the FPU lattices by using this dimensionless parameter $\chi$. Furthermore, with the use of the parameter $\chi$, we can replace the formula of the modulus of the elliptic function $k$ in Eq. (2.25) by

$$
k=\frac{1}{\sqrt{2}} \sqrt{1-\frac{1}{\sqrt{1+4 \chi}}} .
$$

We note here that $k \rightarrow 0$ if and only if $\chi \rightarrow 0$ and this limit can be realized for each set of $\left\{\epsilon, \mu_{2}, \mu_{4} \mid 0<\epsilon, \mu_{2}, \mu_{4}<\infty\right\}$ when we take $n$ (:odd) a fully great number. If we recall that in Theorem 1 it is essential for proving the non-integrability of the FPU lattices to take the limit $k \rightarrow 0(\chi \rightarrow 0)$ so that the eigenvalues of $g_{1}\left(\lambda_{j}\right)$ approach the quantities in Eq. (3.12), we have the following theorem:

Theorem 2 A FPU lattice(2.0) characterized by an arbitrary set of the system parameters $\left\{\epsilon, \mu_{2}, \mu_{4} \mid 0<\epsilon<\infty, 0<\mu_{2}<\infty, 0<\mu_{4}<\infty\right\}$ has no additional analytic integrals of motion besides the Hamiltonian itself for a fully great number of degrees of freedom $n(n: o d d)$. 
This result shows that we need a fully great number $n$ of degrees of freedom to guarantee the strongest non-integrability $\varrho\left(\left\{H_{F P U}, \epsilon\right\}\right)=3^{n-1}$ of each FPU lattice $H_{F P U}$ with the systems parameters $\left\{\mu_{2}, \mu_{4} \mid 0<\mu_{2}<\infty, 0<\mu_{4}<\infty\right\}$ for $0<\epsilon<\infty$ and a problem remains still open as to whether we can prove the non-integrability of each FPU lattices with the system parameters $\left\{\mu_{2}, \mu_{4}, n \mid 0<\mu_{2}<\infty, 0<\mu_{4}<\infty, n\right.$ : odd, $\left.n \geq 3\right\}$ for a given finite energy $\epsilon(0<\epsilon<\infty)$. Clearly, Theorem 1 and Theorem 2 have the reciprocal conditions in terms of the system parameters $\left\{\epsilon, \mu_{2}, \mu_{4}, n\right\}$ and we can unify them into a single theorem as follows:

Theorem 3 A FPU lattice(2.0) characterized by the system parameters $\left\{\epsilon, \mu_{2}, \mu_{4}, n \mid 0<\epsilon<\infty, 0<\mu_{2}<\infty, 0<\mu_{4}<\infty, n \geq 3, n\right.$ : odd $\}$ has no additional analytic integrals of motion besides the Hamiltonian itself when the following condition

$$
\chi=\frac{\epsilon \mu_{4}}{(n+1) \mu_{2}^{2}} \rightarrow 0
$$

is satisfied.

\section{Summary and discussion}

We have got the non-integrability proof of the FPU lattices by tracing the following steps. In Section 2, we have shown that the singularity analysis can be performed towards the non-homogeneous nonlinear lattices by finding the special straight-line solutions in terms of elliptic functions. Especially, it is shown there that the normal variational equations can be decoupled into separated equations and each of them happens to be a Lamé equation and consequently we can compute the eigenvalues of the monodromy matrices associated with the counterclockwise loop along the period cell of the special solutions. In Section 3, with the use of Yoshida's theorem whose validity is guaranteed when that the normal variational equations can be decoupled, we can prove the non-existence of the additional analytic conserved quantities besides the Hamiltonian itself for FPU lattices in the low energy limit. In Section 4, we have considered the classification of FPU lattices via the degree of non-integrability. There, by introducing a transformation $\Re$ from a nonhomogeneous nonlinear lattice into another non-homogeneous nonlinear lattice, which preserves the equations of motion, we have shown that the degree of non-integrability of each FPU lattice in the high energy limit is the same as that of the homogeneous nonlinear lattice whose non-integrability is known to be proven by the analysis using Kowalevski exponents. In case of FPU lattices with arbitrary energy in $0<\epsilon<\infty$, we have a theorem showing the non-integrability for a fully great number of degrees of freedom by introducing a dimensionless parameter which characterizes the normal variational equations. This theorem (Theorem 2) and Theorem 1 have the reciprocal sufficient conditions for the non-integrability of FPU lattices and finally they are unified into a single theorem (Theorem 3 ) by the dimensionless parameter $\chi$.

There remains an open problem to be solved: Can we have a theorem about the non-integrability of more general nonlinear lattices of degree $2 m>4$ ? More specifically speaking, can we have a theorem asserting the non-integrability of the non-homogeneous nonlinear lattices of degree $2 m>4$ such as Theorem 1 and Theorem 2? The present 
neck is in a fact that the normal variational equations along the special solutions of hyper-elliptic functions do not belong to a class of Lamé equations, though they can always be decoupled into $n-1$ separated variational equations, and we cannot compute the eigenvalues of the monodromy matrices associated with these variational equations in general and therefore we cannot perform the Ziglin analysis completely. However, the problem on the degree of non-integrability for more general non-homogeneous nonlinear lattices remains an interesting open question to be solved, because the present analysis on the non-integrability of the FPU lattices, together with Yoshida's argument about the homogeneous nonlinear lattice in terms of Kowalevski exponents, strongly suggests universality about the non-integrability of more general non-homogeneous nonlinear lattices in Eq. (4.4).

\section{Appendix}

In this appendix, we show the difference between the eigenvalues of $g_{*}\left(\lambda_{j}\right)$ and the eigenvalues of $g_{1}^{2}\left(\lambda_{j}\right)$ for any $j\left(\neq \frac{n+1}{2}\right)$. We prove this by reductio ad absurdum. First, we assume that the following equality

$$
\begin{aligned}
& \operatorname{Spec}\left(g_{1}^{2}\left(\lambda_{j}\right)\right) \equiv\left\{\operatorname { e x p } \left(i \pi\left(2 \sqrt{2} \sin \left(\frac{j \pi}{2(n+1)}\right)\right), \exp \left(-i \pi\left(2 \sqrt{2} \sin \left(\frac{j \pi}{2(n+1)}\right)\right)\right\}\right.\right. \\
& =\left\{-\exp \left(\pi i \sqrt{25-24 \cos \frac{j \pi}{n+1}}\right),-\exp \left(-\pi i \sqrt{25-24 \cos \frac{j \pi}{n+1}}\right)\right\} \equiv \operatorname{Spec}\left(g_{*}\left(\lambda_{j}\right)\right)
\end{aligned}
$$

would hold for some $j\left(\neq \frac{n+1}{2}\right)$. Our purpose here is to show that the relation (6.1) is false. By (6.1), one of the following equalities

$$
\begin{aligned}
& \sqrt{25-24 \cos \frac{j \pi}{n+1}}+1+2 m_{+}=2 \sqrt{2} \sin \left(\frac{j \pi}{2(n+1)}\right) \\
& \sqrt{25-24 \cos \frac{j \pi}{n+1}}+1+2 m_{-}=-2 \sqrt{2} \sin \left(\frac{j \pi}{2(n+1)}\right)
\end{aligned}
$$

holds for $m_{+}, m_{-} \in \boldsymbol{Z}$ and $j \neq \frac{n+1}{2}$. Because of the inequalities

$$
1<\sqrt{25-24 \cos \frac{j \pi}{n+1}}<7, \quad 0<2 \sqrt{2} \sin \left(\frac{j \pi}{2(n+1)}\right)<2 \sqrt{2},
$$

it is sufficient to consider the equalities (6.2) and (6.3) for $m_{+} \in\{-3,-2,-1,0\}$ and $m_{-} \in\{-5,-4,-3,-2\}$. If we rewrite $\sin \left(\frac{j \pi}{2(n+1)}\right)$ as $X$, Eq. (6.2) and Eq. (6.3) are changed into the following equations

$$
\begin{aligned}
& 40 X_{+}^{2}+\left(1+2 m_{+}\right) 4 \sqrt{2} X_{+}+1-\left(1+2 m_{+}\right)^{2}=0 \\
& 40 X_{-}^{2}-\left(1+2 m_{-}\right) 4 \sqrt{2} X_{-}+1-\left(1+2 m_{-}\right)^{2}=0
\end{aligned}
$$


respectively. By considering $X\left(=X_{+}, X_{-} \geq 0\right.$, we can easily obtain a series of the solutions as follows:

$$
\begin{aligned}
& X_{+}\left(m_{+}=0\right)=0 \\
& X_{+}\left(m_{+}=-1\right)=\frac{\sqrt{2}}{10} \\
& X_{+}\left(m_{+}=-2\right)=\frac{1}{\sqrt{2}} \\
& X_{+}\left(m_{+}=-3\right)=\frac{5 \sqrt{2}+\sqrt{290}}{20}>\frac{7+\sqrt{289}}{20}=\frac{6}{5}>1 \\
& X_{-}\left(m_{-}=-2\right)=\frac{\sqrt{2}}{5} \\
& X_{-}\left(m_{-}=-3\right)=\frac{-5 \sqrt{2}+\sqrt{290}}{10} \\
& X_{-}\left(m_{-}=-4\right)=\frac{1}{\sqrt{2}} \\
& X_{-}\left(m_{-}=-5\right)=\frac{-9 \sqrt{2}+\sqrt{962}}{20} .
\end{aligned}
$$

Furthermore, because of the following relations $0<X_{ \pm}<1, \quad X_{ \pm} \neq \frac{1}{\sqrt{2}}$ for $j \neq \frac{n+1}{2}$, we can discard the solutions $X_{+}\left(m_{+}=0\right), X_{+}\left(m_{+}=-2\right), X_{+}\left(m_{+}=-3\right), X_{-}\left(m_{-}=-4\right)$. For the solutions $X_{+}\left(m_{+}=-1\right), X_{-}\left(m_{-}=-2\right)$, we have the equalities as

$$
\begin{aligned}
& \cos \frac{j \pi}{n+1}=1-2\left(X_{+}\left(m_{+}=-1\right)\right)^{2}=\frac{24}{25} \\
& \cos \frac{j \pi}{n+1}=1-2\left(X_{-}\left(m_{-}=-2\right)\right)^{2}=\frac{21}{25}
\end{aligned}
$$

respectively. However, these relations (6.8) contradict with a fact [11] that

$$
\cos \frac{j \pi}{n+1} \in \boldsymbol{Q} \Longleftrightarrow \cos \frac{j \pi}{n+1}=0, \quad \text { or } \quad \frac{1}{2}, \quad \text { or } \quad-\frac{1}{2} \text {. }
$$

Thus, we can also discard the solutions $X_{+}\left(m_{+}=-1\right)$ and $X_{-}\left(m_{-}=-2\right)$. Let us consider the solutions $X_{-}\left(m_{-}=-3\right)$ and $X_{-}\left(m_{-}=-5\right)$. If we rewrite $\exp \frac{j \pi}{n+1}$ as $\zeta$, the following relation

$$
25 \zeta^{4}+70 \zeta^{3}-46 \zeta^{2}+70 \zeta+25=0
$$

is satisfied for the solution $X_{-}\left(m_{-}=-3\right)$, while the relation

$$
25 \zeta^{4}+462 \zeta^{3}+626 \zeta^{2}+462 \zeta+25=0
$$

is satisfied for the solution $X_{-}\left(m_{-}=-5\right)$. Since $\zeta$ is one of $2(n+1)$-th root of unity and $2(n+1) \quad(\bmod 4)=0$, the minimal polynomial $P(\zeta)$ with coefficients of rational integers which has a solution $\zeta$ for $P(\zeta)=0$ must be one of the cyclotomic polynomials $\Phi_{l}(Y) \in Z[Y]:$

$$
\Phi_{l}(Y)=\prod_{i=1}^{\varphi(l)}\left(Y-\zeta_{i}\right),
$$

where $2(n+1)(\bmod l)=0,\left\{\zeta_{i}\right\}$ is a set of all primitive $l$ th roots of unity and $\varphi(l)$ is the number of positive integers which are less than or equal to $l$ and relatively prime to $l$ and this function $\varphi$ is called Euler's $\varphi$-function. All cyclotomic polynomials $\Phi_{l}(Y)$ are known to be irreducible over $\boldsymbol{Q}$ and we can check that cyclotomic polynomials $\Phi_{l}(Y)$ whose degrees are less than or equal to 4 are restricted to the case 12 that

$$
\begin{aligned}
& \aleph \equiv\left\{\Phi_{l}(Y) \mid 2 \leq \operatorname{Deg} \Phi_{l}(Y) \leq 4\right\} \\
& =\left\{\Phi_{3}(Y)=Y^{2}+Y+1, \Phi_{4}(Y)=Y^{2}+1, \Phi_{5}(Y)=Y^{4}+Y^{3}+Y^{2}+Y+1,\right. \\
& \left.\Phi_{6}(Y)=Y^{2}-Y+1, \Phi_{8}(Y)=Y^{4}+1, \Phi_{12}(Y)=Y^{4}-Y^{2}+1\right\}
\end{aligned}
$$


Using this fact, we can say that a cyclotomic polynomial $\Phi_{l}(Y) \in \aleph$ must divide either the polynomial

$$
P_{1}(Y) \equiv 25 Y^{4}+70 Y^{3}-46 Y^{2}+70 Y+25
$$

in Eq.(6.10) or the polynomial

$$
P_{2}(Y) \equiv 25 Y^{4}+462 Y^{3}+626 Y^{2}+462 Y+25
$$

in Eq.(6.11). It is easy to check that this is impossible. Thus now, we can conclude that the assumption (6.1) is false; i.e., we have shown that for any $j\left(\neq \frac{n+1}{2}\right)$

$$
\operatorname{Spec}\left(g_{1}^{2}\left(\lambda_{j}\right)\right) \neq \operatorname{Spec}\left(g_{*}\left(\lambda_{j}\right)\right) .
$$

\section{Acknowledgements}

The majority of this work was carried out to complete the doctoral dissertation at Department of Physics, University of Tokyo. I would like to thank Prof. Masuo Suzuki, Prof. Miki Wadati and Prof. Haruo Yoshida for valuable discussions. While writing this work up I have been supported from the Special Researcher's Program towards Basic Science at the RIKEN and from the Program of the Complex Systems II at the International Institute for Advanced Study (IIAS-Kyoto). I would like to thank Prof. Shun-ichi Amari for his continual encouragement. 


\section{References}

[1] P. F. Byrd and M. D. Friedman, Handbook of elliptic integrals for engineers and scientists (Springer, New York, 1971).

[2] R. C. Churchill and D. L. Rod, "Geometrical aspects of Ziglin's non-integrability theorem for complex Hamiltonian systems", J. Diff. Eq. 76(1988):91-114.

[3] E. Fermi, J. Pasta and S. Ulam, Los Alamos Report LA-1940(1955); in: Analogies between Analogies: the mathematical reports of S.M. Ulam and his Los Alamos collaborators, Edited by A.R. Bednarek and F. Ulam, (Univ. of California Press, 1990).

[4] A. Hejhal, "Monodromy groups and linearly polymorphic functions", Acta Math. 135(1975):1-55.

[5] M. Hénon and C. Heiles, The applicability of the third integral of motion; some numerical experiments, Astoronom. J 69(1964):73-79.

[6] H. Ito, "Non-integrability of Hénon-Heiles system and a theorem of Ziglin", Kodai Math. J. 8(1985):120-138.

[7] W. Magnus and S. Winkler, Hill's Equation, (Dover, New York, 1979).

[8] H. Poincaré, Les Nouvelles Méthodes de la Mécanique Céleste Tome. I (Paris:Gauthier-Villars, Paris, 1892).

[9] A. Ramani, B. Grammaticoss and T. Bountis, "The Painlevé property and singularity analysis of integrable and non-integrable systems", Phys. Rep. 180(1989):159245.

[10] K. Takano and E. Bannai, " A global study of Jordan-Pochhammer differential equations", Funkcialaj Ekvaciaj 19(1976):85-99.

[11] K. Umeno, "Galois extensions in Kowalevski exponents and nonintegrability of nonlinear lattices", Phys. Lett. A 190(1994):85-89.

[12] K. Umeno,Non-integrability proof in some Hamiltonian dynamical systems with three or more degrees of freedom, doctoral dissertation, (University of Tokyo, 1994, December).

[13] K. Umeno, "Non-integrable character of Hamiltonian systems with global and symmetric coupling", Physica D 82(1995):11-35.

[14] E. T. Whittaker, Analytical Dynamics, (Cambridge Univ. Press, 1904), chap.14.

[15] E. T. Whittaker and G. N. Watson, A Course of Modern Analysis, (Cambridge Univ. Press, London/New York, 1969). 
[16] H. Yoshida, "A criterion for the non-existence of an additional integral in Hamiltonian systems with a homogeneous potential", Physica D 29 (1987):128-142.

[17] H. Yoshida," A criterion for the non-existence of an additional analytic integral in Hamiltonian systems with n degrees of freedom", Phys. Lett. A 141(1989):108-112.

[18] H. Yoshida," Necessary condition for the existence of algebraic first integrals I: Kowalevski's Exponents", Celestial Mechanics 31(1983):363-379.

[19] S. L. Ziglin," Branching of solutions and non-existence of first integrals in Hamiltonian mechanics. I.", Funct. Anal. Appl. 16(1983):181-189.

[20] S. L. Ziglin, "Branching of solutions and non-existence of first integrals in Hamiltonian mechanics. II."17(1983):6-17. 